\title{
Conjuntura Austral
}

\section{MINERÍA PARA EL DESARROLLO INTEGRAL EN LA ESTRATEGIA DE UNASUR}

\section{A UNASUR strategy for mining and development}

\author{
Marcelo Saguier ${ }^{1}$
}

\section{Introducción}

La región sudamericana cuenta con las mayores reservas petroleras, acuíferas y de biodiversidad del mundo. En un contexto global en el que estos recursos están en gran demanda, la capacidad de los países de la región para coordinar con autonomía sus políticas en materia de recursos naturales adquiere especial relevancia estratégica. De ello dependen las posibilidades de ejercer soberanía sobre los recursos, condición necesaria para impulsar políticas de desarrollo integral. Es en base a tal diagnóstico que los gobiernos de la región promueven en el marco de la Unión de Naciones Sudamericanas (UNASUR) una Estrategia Integral para el Aprovechamiento Conjunto de los Recursos Naturales, iniciativa anunciada encumbre presidencial de Lima de 2012 .

El proceso UNASUR incorpora las políticas sobre recursos naturales desde su origen. En la cumbre presidencial de Margarita de 2007 se acuerda la creación del bloque regional y simultáneamente se propone la negociación de un Tratado Energético Sudamericano. El posterior llamado a diseñar una estrategia sobre recursos naturales busca dinamizar la agenda regional energética, ya sin el impulso que supo imprimir el gobierno de Hugo Chávez de Venezuela en lo referido a hidrocarburos, pero también

\footnotetext{
${ }^{1} \mathrm{PhD}$ en Política y Estudios Internacionales (University of Warwick), Reino Unido. Investigador Principal Área de Relaciones Internacionales FLACSO/Argentina. Miembro del Consejo de Investigaciones Científicas y Técnicas (CONICET). E-mail: msaguier@ flacso.org.ar.
} 


\section{Conjuntura Austral}

incorporar a la minería como un sector crecientemente considerado estratégico por los gobiernos de la región.

El compromiso de formular una perspectiva regional de los recursos naturales para el desarrollo integral se inscribe en una concepción política de regionalismo "posthegemónico" (Riggirozzi y Tussie, 2012) o "post-neoliberal” (Rodríguez-Garavito et al., 2008) que emergede la crisis del paradigma neoliberal de la integración. Si bien tales caracterizaciones no están exentas de controversia, el fracaso del proyecto Área de Libre Comercio de las Américas (ALCA) en el 2005 es el punto de inflexión en el contenido y los procesos que rigen la integración en la región. La creación de la UNASUR cristaliza un nuevo momento en las relaciones internacionales de Sudamérica, caracterizado por la afirmación de una identidad común como parte de una comunidad política regional. Este proceso permitió el desarrollo de un espacio de convergencia política para la resolución de conflictos diplomáticos, resguardo del orden democrático, solidaridad y búsqueda de convergencia para la definición coordinada de políticas nacionales - de defensa, infraestructura, salud, políticas sociales, desarrollo social.

En el plano de las políticas de recursos naturales, el actual movimiento regionalista plantea,por un lado, la afirmación de la soberanía de los estados sobre los recursos y, por otro, una concepción de los recursos naturales como bienes comunes a ser administrados en forma conjunta por los países de la región en una suerte de soberanía de condominio. Lo segundo supone una redefinición de la mirada tradicionalmente estatista de la soberanía. No se postula aquí una relación antagónica o excluyente entre concepciones estatista-territoriales y post-territoriales o postnacionales (Sassen, 2006). Por el contrario, se propone un enfoque que considera ambos conceptos como procesos dinámicos, recíprocamente constitutivos y constituyentes. En otras palabras, las posibilidades de construir un horizonte común en la UNASUR para la coordinación de políticas nacionales de recursos naturales dependen de la rearticulación del campo discursivo "nacional" - como espacio definido por su diferencia de la alteridadde otros estados-nación - a partir de la apropiación de un discurso "regional" como espacio ordenador y fundante. Ello plantea la importancia de la UNASUR como 


\section{Conjuntura Austral}

plataforma para articular y proyectar nuevas miradas sobre los recursos naturales y el desarrollo integral que incidan en el cambio de percepciones nacionales.

Sin embargo, las posibilidades de formularde una estrategia continental de los recursos naturales en un contexto regional marcadamente heterogéneo son al menos inciertas. Las diferencias de visiones y estrategias de inserción internacional de los países del bloque difieren substancialmente. Por un lado, los que integran la Alianza del Pacífico se orientan a la especialización en materias primas y son signatarios de Tratados de Libre Comercio (TLCs) que restringen el espacio político de los estados para modificar o proponer políticas - cuando las empresas consideran que sus intereses son afectados. Por otro, los países del Mercosur, especialmente Brasil y Argentina, promueven la diversificación productiva mediante el desarrollo industrial - sibien el neo-extractivismo como modelo de acumulación es también una tendencia entre gobiernos progresistas con las implicancias reprimarización económica que ello supone (Gudynas, 2012).

A esto se sumala ausencia de consensos sociales acerca del lugar que ocupan, o deberían ocupar, los recursos naturales/bienes naturales en las expectativas de "desarrollo" en cada sociedad. La crisis del neoliberalismo condujo a una repolitización de los recursos naturales (Hogenboom, 2012). La ausencia de tales consensos se manifiesta en la proliferación de conflictos socio-ambiental esvinculados a recursos naturales en toda la región (Bebbington, 2012; OLCA, 2013; Saguier, 2012a; 2012b). Es por ello que la aspiración de que la UNASUR contribuya a la definición del contenido de una visión compartida sobre el "desarrollo integral" es ciertamente una meta ambiciosa. Por último, a esto se suma la diversidad de ordenamientos jurídicos nacionales sobre recursos naturales que dificulta el aunar criterios comunes de políticas (OLADE, 2013).

En un contexto regional con tales características socio-políticas e institucionales surge el interrogante de si es viable alcanzar una estrategia UNASUR de aprovechamiento conjunto de los recursos naturalespara el desarrollo integral.¿En qué condiciones podría ser viable? Para responder a estos interrogantes el artículo focaliza 


\section{Conjuntura Austral}

en la minería como uno de los recursos naturales sujetos a la estrategia UNASUR. Quedan fuera del foco del artículo los hidrocarburos, la hidro-energía y la biodiversidad.

El argumento aquí propuesto es que UNASUR constituye una oportunidad para una estrategia regional sobre políticas de minería solo en la medida que los gobiernos sean capaces de cooperar para el fortalecimiento de las capacidades del estado de intervenir en el mercado de minerales, regular la actividad minera y generar condiciones para democratizar las políticas mineras. Si ello no ocurriera, significaría que el proceso UNASUR correría es riesgo de viabilizar políticas extractivistas de minería mediante la cooperación regional. Esto no solo llevaría al fracaso del intento por formular una mirada común sobre el desarrollo integral, sino que debilitaría a la región en su conjunto en su capacidad de incidir y administrar su inserción internacional en un mundo con nuevas correlaciones de fuerza.

En la primera sección se fundamenta porqué la estrategia UNASUR es importante estratégicamente en términos de construcción regional yde posicionamiento internacional del bloque. En las secciones subsiguientes se identifican algunas áreas de política en torno a los cuales articular el debate regional sobre un marco de gobernanza de recursos naturales.

\section{La relevancia de una estrategia regional sobre recursos naturales para el desarrollo integral}

La iniciativa de la UNASUR consiste en una estrategia de cooperación que busca aumentar los márgenes de autonomía política de los estados miembro para hacer frente a los desafíos y oportunidades que plantea la reconfiguración de la estructura de poder internacional. La nueva configuración de poder internacional está signada por dos procesos simultáneos, de transferencia y de difusión del poder internacional (Keohane y Nye, 2002).

El primero es la transferencia de poder de los países centrales hacia los países con economías emergentes del Sur. La alianza BRICS y la iniciativa IBSA son solo algunas de las nuevas articulaciones de la cooperación Sur-Sur que redefinen el campo de la política mundial. Ello no implica necesariamente la declinación los antiguos 


\section{Conjuntura Austral}

centros de poder en los EEUU y Europa, aunque si su reformulación en un contexto en el que éstos requieren negociar su posición internacional en redes de interdependencia económica, política y social con los países emergentes. El G-20 surge como nueva plataforma de gobernanza global para dar cuenta del nuevo equilibrio de poder e intento de compartir responsabilidades entre un numero mayor de actores. La cooperación en políticas para la minería en la UNASUR tiene como desafío asegurar convergencia regional que permita posicionamientos comunes frente a los principales demandantes de minería y exportadores de inversión minera en la región - China, Canadá, Australia, Reino Unido, Suiza, etc.

El segundo proceso de cambio en la estructura de poder internacional consiste en la difusión del poder de los estados hacia actores no-estatales, fundamentalmente a las empresas transnacionales (ETNs), aunque también a actores sociales como organizaciones de la sociedad civil y movimientos sociales transnacionales.

El poder internacional de las ETNs se expresa en tres dimensiones indisociables: instrumental, estructural y discursiva (Fuchs, 2004). La dimensión instrumental del poder, a través de su capacidad de lobby en el estado y en organismos internacionales. Esto se ve favorecido por la enorme disponibilidad de recursos económicos y técnicos del que disponen las empresas para el lobby, y por las recurrentes prácticas de "puertas giratorias" que relativizan la distinción entre funcionarios públicos y empresas.

La dimensión estructural del poder de las ETNs se relaciona con su control oligopólico en el mercado, que se traduce en la capacidad para instalar y excluir temas de agenda, además de instigar la competencia regulatoria entre los estados. En materia regulatoria, las ETNs promueven regímenes de auto-regulación voluntaria (estándares, códigos de conducta e iniciativas de responsabilidad social corporativa) en contraposición a los de carácter obligatorio y vinculante o incluso a esquemas híbridos (Utting, 2008).

La dimensión discursiva del poder de las ETN sconsiste en la capacidad para construir legitimidad, en tanto actores políticos involucrados en la toma de decisión, definición de reglas y auto-regulación, mediante la articulación de narrativas que definen la identidad de la empresa como garante del bien público. En muchos casos, la 


\section{Conjuntura Austral}

coordinación discursiva de las empresas es parte de estrategias de cooperación global entre firmas que establecen parámetros comunes para la segmentación del mercado y un funcionamiento sectorial transnacionalmente coordinado de acuerdo a niveles de institucionalización variable (Cutler, 2003). En el campo de la minería, la iniciativa del Consejo Internacional de Minería y Metales (ICMM por su sigla en inglés), organización liderada por directores ejecutivos de la industria, incide en el definición de visiones del "desarrollo sustentable" afines a las prioridades de la industria.

Los movimientos sociales y organizaciones de la sociedad civil emergen también como actores de poder internacional. A través del activismo transnacional establecen coaliciones de actores sociales en función de: campañas regionales y globales, generación conjunta de información, estrategias de incidencia política en ámbitos nacionales e internacionales, coordinación discursiva, así como La diseminación, reinterpretación y construcción de normas internacionales (Keck y Sikkink, 1998; Saguier, 2007; Tarrow, 2005). En América latina, la minería es un punto focal de convergencia de activismo transnacional, particularmente mediante el Observatorio de Conflictos Mineros de América Latina (OCMAL). Integrado por 40 organizaciones sociales de la región, OCMAL indaga en las contradicciones entre mega-minería y desarrollo en términos ecológicos, de derechos humanos y colectivos y propone reformas a los modelos mineros vigentes en la región. La Coordinadora Andina de Organizaciones Indígenas (CAOI) es otro espacio de articulación transnacional de relevancia. Estos espacios funcionan como dinámicas de "regionalismo desde abajo" redefiniendo el campo de discusión en ámbitos nacionales en lo relacionado a la relación entre derechos y recursos naturales.

La influencia de nuevos actores estatales, empresariales y sociales en las dinámicas de poder internacional demanda miradas que sean capaces de articular políticas multidimensionales. La UNASUR puede actuar como un espacio político catalizador de estas miradas, instrumentos de política y alianzas para orientar el horizonte acción de los gobiernos en un mundo en transición desde un lugar de autonomía política y desarrollo integral. Los riesgos de no hacerlo es que la soberanía de los estados sobre los recursos naturales termine siendo un formalismo legalista 


\section{Conjuntura Austral}

carente de contenido y prácticas políticas y sociales legítimas que le den sustento. La gobernanza regional de los recursos naturales para el desarrollo es tanto una oportunidad para construcción de cohesión y desarrollo interno en la región, como para la autonomía del bloque en un contexto global cambiante. De ello depende lograr superar la histórica dependencia como periferia proveedora de recursos naturales, ya no sólo a los centros de poder conformado por países industrializados del Norte, sino también a los nuevos poderes emergentes del Sur.

A continuación se identifican algunas áreas de política para estimular el incipiente diálogo regional en torno a enfoques cooperativos para la minería y el desarrollo integral. La selección de temas no se pretende exhaustiva.

\section{Generación y centralización de información geológica}

Ejercer la soberanía sobre los recursos naturales supone aumentar las capacidades de los estados para generar y disponer de información geológica. Esta información es fundamental para poder elaborar diagnósticos de políticas de desarrollo que involucren al sector minero.

La información geológica sobre la presencia y ubicación geográfica de yacimientos de minerales es generada mayoritariamente por empresas privadas dedicadas a la exploración. Estas cuentan con expertise técnico y capacidad tecnológica que les permite ofrecer servicios de información y asesoramiento a grandes ETNs dedicadas a la explotación de los minerales. La Geological Survey de los Estados Unidos (USGS), dependiente del Departamento del Interior de ese país, es también un actor importante en la generación de información geológica vinculado a las ETNs extractivas como proveedor de servicios de información y asesoramiento. América latina y el Caribe en actualmente el principal destino de la inversión en exploración minera mundial. Los presupuestos de exploración en la región se han multiplicado más de cinco veces entre 2003 y 2010, pasando de 566 millones a 3.024 millones de dólares anuales. La exploración se ha concentrado principalmente en Perú, México, Brasil, Chile y en menor medida en Argentina (CEPAL, 2013: 26-28). 


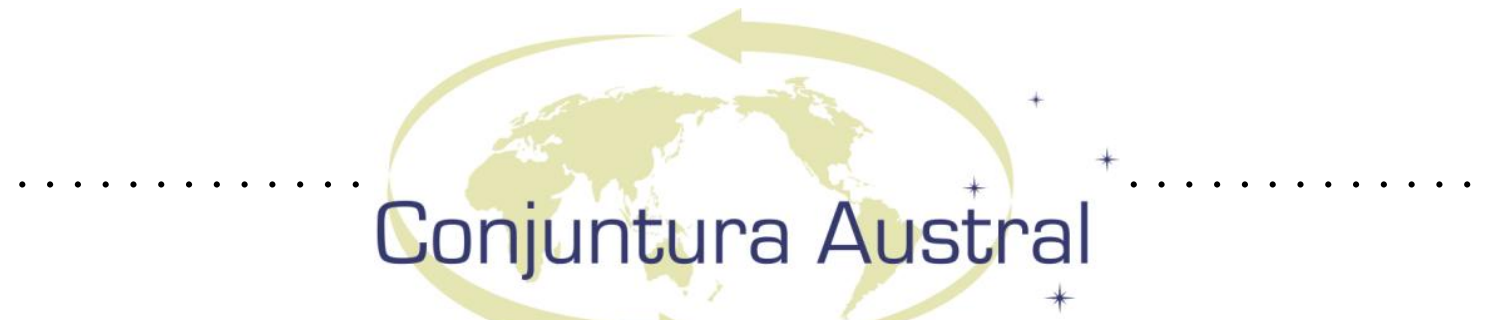

La capacidad de generar y disponer de información geológica incide favorablemente en las condiciones de negociación de los estados con empresas privadas - para acuerdos sobre compromisos y condiciones de inversión, para dimensionar el rendimiento de los proyectos mineros y las utilidades de las empresas a los fines de definir prospectivamente planes de inversión pública en base a ingresos prevenientes de la minería, etc. Sin esta información los estados carecen de elementos para formular políticas.

El reconocimiento del valor estratégico de la información geológica dio lugar a la Conferencia de Recursos Naturales y Desarrollo Integral de la UNASUR realizada en Caracas del 27 al 30 de mayo 2013. El evento recogió opiniones de 40 investigadores $^{2}$ y culminó con la propuesta de confeccionar un inventario integrado de los recursos naturales con los que cuenta la UNASUR. Para ello se propuso la creación de un Centro de Estudios Avanzados sobre los recursos naturales de la región, un Servicio Especial Geológico y una red de conocimiento y acercamiento de UNASUR a las redes de investigación.

La información geológica es sin duda indispensable para mejorar las condiciones de planificación y gestión al estado de los recursos mineros, remitiendo al ámbito público competencias que se encuentran en las empresas privadas. Sin embargo, la iniciativa de regionalizar esta información en la UNASUR no asegura por si sola que la producción minera esté inserta en una visión de desarrollo integral. La construcción política de esta mirada es esencial para evitar que el fortalecimiento de las capacidades de los estados para diagnosticar sus recursos mineros legitime y profundice la actual tendencia neo-extractivista y predatoria de los recursos.

\section{Regímenes fiscales: reformas coordinadas o carreras regulatorias}

La participación del estado en la renta de la actividad minera es importante para asegurar la solvencia fiscal de los países. Esto no supone que la minería represente la

\footnotetext{
${ }^{2}$ Los aportes se enmarcaron en los siguientes ejes temáticos: recursos naturales y soberanía; recursos naturales y ambiente; recursos naturales y desarrollo científico; la necesidad de incorporar las visiones de las comunidades indígenas y campesinos; y recursos naturales y fondos de financiamiento.
} 


\section{Conjuntura Austral}

principal actividad económica, aunque es cuestionable que la misma no ocupara lugar alguno en las estrategias de desarrollo de los países.

El aumento del precio internacional de los minerales ha reabierto el debate sobre las condiciones de la participación del estado en la renta minera. Desde el 2003 a la fecha ha habido un aumento del $300 \%$ en el precio internacional de los minerales (Heidrich, 2013). Los aumentos de los precios se deben a la demanda de China de minerales y otros bienes primarios, producto de su proceso de industrialización y crecimiento. Asimismo, la especulación del mercado financiero de minerales es otro factor del aumento de los precios. Esto se ha reflejado en un aumento de la renta económica de las actividades mineras como porcentaje del PBI de los países. Entre 2004 y 2009 llegó casi a cuadruplicarse con respecto al promedio del período 1990-2003, al pasar del 0,54\% al 2,08\% del PIB de los países de América Latina y el Caribe (CEPAL, 2013: 31).

Ante el aumento de precios los gobiernos de la región comenzaron a incursionar en la minería con la creación de empresas estatales. Ecuador crea la Empresa Nacional Minera de Ecuador (Enami) en el 2010 y se asocia a la chilena Codelco para la exploración del cobre. Por su parte, Bolivia funda la en el 2012 la Empresa Minera Coro Coro (EMC), dependiente de la Corporación Minera de Bolivia (COMIBOL), con el objetivo de incrementar y mejorar la cadena productiva integral del cobre en el país. El establecimiento en Argentina de la Organización Federal de Estados Mineros (OFEMI) en 2012 impulsó la creación de diez empresas mineras provinciales con las que se cristaliza un nuevo arreglo institucional federal para la producción minera y distribución de la renta entre estado nacional y provincias.

No obstante el aumento de esta renta minera en términos absolutos (como ingreso fiscal), los regímenes fiscales de los países de la región no cuentan con criterios de progresividad en las escalas de tributación minera. Esto se debe a que los regímenes tributan en base a regalías (o royalties), la forma más común de tributo utilizado para la industria minera en América Latina y el mundo. En la mayoría de los países latinoamericanos hubo una reducción del porcentaje de royalties durante la década del 80 y 90 como parte de las recomendaciones del Banco Mundial y el Fondo Monetario 


\section{Conjuntura Austral}

Internacional de acuerdo a la visión neoliberal imperante en la época (los royalties bajaron en promedio entre el 10-15\% a 1-5\%). Cuando estas políticas eran implementadas los precios internacionales de los minerales se encontraban extremadamente bajos, y por ello que no se esperaba que la minería contribuyera significativamente como fuente de ingresos fiscales. En cambio, la prioridad de los gobiernos era instaurar marcos institucionales favorables a la radicación de inversión extranjera directa (CEPAL, 2013: 29; Heidrich, 2013).

Para avanzar en criterios de progresividad en la tributación es preciso poder determinar cuál es la rentabilidad de las empresas, la cual se ha incrementado significativamente. Los impuestos en mayoría de los países están basados en la utilidad declarada de las empresas, lo que habilita a prácticas de ocultamiento de utilidades, evasión y al aumento artificial de costos por parte de las empresas. Difícilmente puedan los estados fiscalizar a las empresas si los márgenes institucionales para la evasión no son modificados para transparentar rentabilidades y costos del sector (CEPAL, 2013: 90).

Algunos países han implementado reformas en años recientes, aunque en forma descoordinada con otros países de la región. Perú incorporo en 2011 un nuevo esquema de regalías que incorpora un impuesto adicional de más de $34 \%$ en base a las ganancias. El parlamento uruguayo acaba de aprobar una ley minera en septiembre de 2013 que fija impuestos de hasta $50 \%$ a las ganancias de los emprendimientos variable a $60 \%$ en caso que suban los precios internacionales. ${ }^{3}$ Por su parte, el congreso brasileño trata un nuevo código minero propuesto por el gobierno en el que se busca incrementar las regalías en base a ingresos brutos que se cobran al sector hasta un 4\% (lo que representa el doble de lo actual) además de introducir un esquema de distribución de las regalías en el que los municipios productores recibirán 65\%, los estados $23 \%$ la Unión Federal 12\%. Asimismo, México discute en una ley para subir las regalías de 5 a 7,5\%, mientras que la República Dominicana renegoció con la minera canadiense Barrick

\footnotetext{
${ }^{3}$ http://www.paisminero.co/index.php?option=com_content\&view=article\&id=11946:uruguay-apruebaley-de-megamineria-y-apunta-a-diversificar-su-matriz-productiva\&catid=117:mineriacolombiana\&Itemid $=499$.

${ }^{4}$ http://www.mme.gov.br/sgm/menu/marco_regulatorio/marco_regulatorio_da_mineracao.html
} 


\section{Conjuntura Austral}

Gold en mayo del 2013 un aumento el canon de 3 a 18\%. Estos antecedentes sugieren nuevos márgenes de maniobra con los que cuentan los estados para impulsar un debate regional sobre reformas de regímenes fiscales que incorporen gravámenes a las ganancias de las empresas.

Las empresas no se han mantenido pasivas frente al ciclo de reformas. Barrick Gold amenazó al gobierno dominicano en demandar ante los tribunales del Centro Internacional de Arreglo de Diferendos e Inversiones (CIADI), el tribunal arbitral del Banco Mundial, e inclusive en retirarse del país dejando un tendal de desocupados y de empresas proveedoras al borde de la bancarrota si el gobierno insistía con aumentar los impuestos. Finalmente la empresa aceptó un nuevo acuerdo. Las empresas propician la competencia regulatoria entre los distintos países del bloque en el debate regional sobre reforma tributaria. Los regímenes tributarios de minería privada en Chile y Perú son presentados como modelos insignia para incentivar la radicación de inversiones y mejorar competitividad del sector, inclusive con propuestas de cambio de políticas tributarias, salariales y ambientales, cambiarias y de comercio internacional afines a generar condiciones de competitividad (MiningPress, 2013).

UNASUR ofrece un espacio donde comenzar a intercambiar experiencias y propuestas para reformar los regímenes fiscales de la minería con criterios de progresividad y mecanismos efectivos de fiscalización. El contexto económico y político regional es favorable para ello. La cooperación es indispensable para contener las tendencias de empresas mineras a generar un clima de competencia regulatoria entre los países. Esto erosionaría las posibilidades de ejercer la soberanía de los estados sobre sus recursos naturales.

\section{Alianzas minerales para política de precios}

La estabilidad de los precios de los minerales en el mercado internacional es condición necesaria para que los países exportadores de la UNASUR puedan planificar políticas de desarrollo integral de largo plazo.

Las cadenas de valor de la minería a escala global están concentradas en un grupo reducido de ETNs de Estados Unidos, Canadá y Australia. Inversiones mineras 


\section{Conjuntura Austral}

chinas son cada vez más importantes también, especialmente en Ecuador y Perú. La posición oligopólica de la industria minera en términos globales le confiere a las grandes empresas capacidad para incidir en los precios internacionales regulando la inversión, volumen y condiciones de la producción. No obstante ello, los países de la UNASUR cuentan con condiciones para aumentar su influencia para incidir en los precios internacionales de los minerales.

En primer lugar, algunos de estos países son líderes de producción en términos globales. Trece países de América Latina ocupan una posición dentro de los 15 mayores productores de minerales del mundo. Chile lidera como el productor mundial de cobre, Brasil se encuentra entre los tres productores principales de hierro, Perú es uno de los primeros productores mundiales de plata, cobre, oro y plomo, en tanto que Bolivia es el cuarto productor de estaño de mina y el sexto de plata, mientras que Colombia es el séptimo productor de níquel refinado. La producción minera de la región "es tan importante a escala mundial que, cuando hay expectativas de suspensiones temporales de operaciones o cuando realmente sucede algún problema interno en alguna gran empresa minera latinoamericana (huelgas o accidentes, por ejemplo), la cotización de los minerales se ve afectada en las principales bolsas de los mercados internacionales" (CEPAL, 2013: 21).

En segundo lugar, los países de la UNASUR disponen conjuntamente una parte considerable de las reservas de algunos minerales en términos globales. Ello les confiere ventaja estratégica con respecto a la posibilidad de aunar criterios en base a pautas de producción de minerales con mayor concentración en la región. 


\section{Conjuntura Austral}

\section{CUADRO 1: Reservas de minerales comparadas con el mundo}

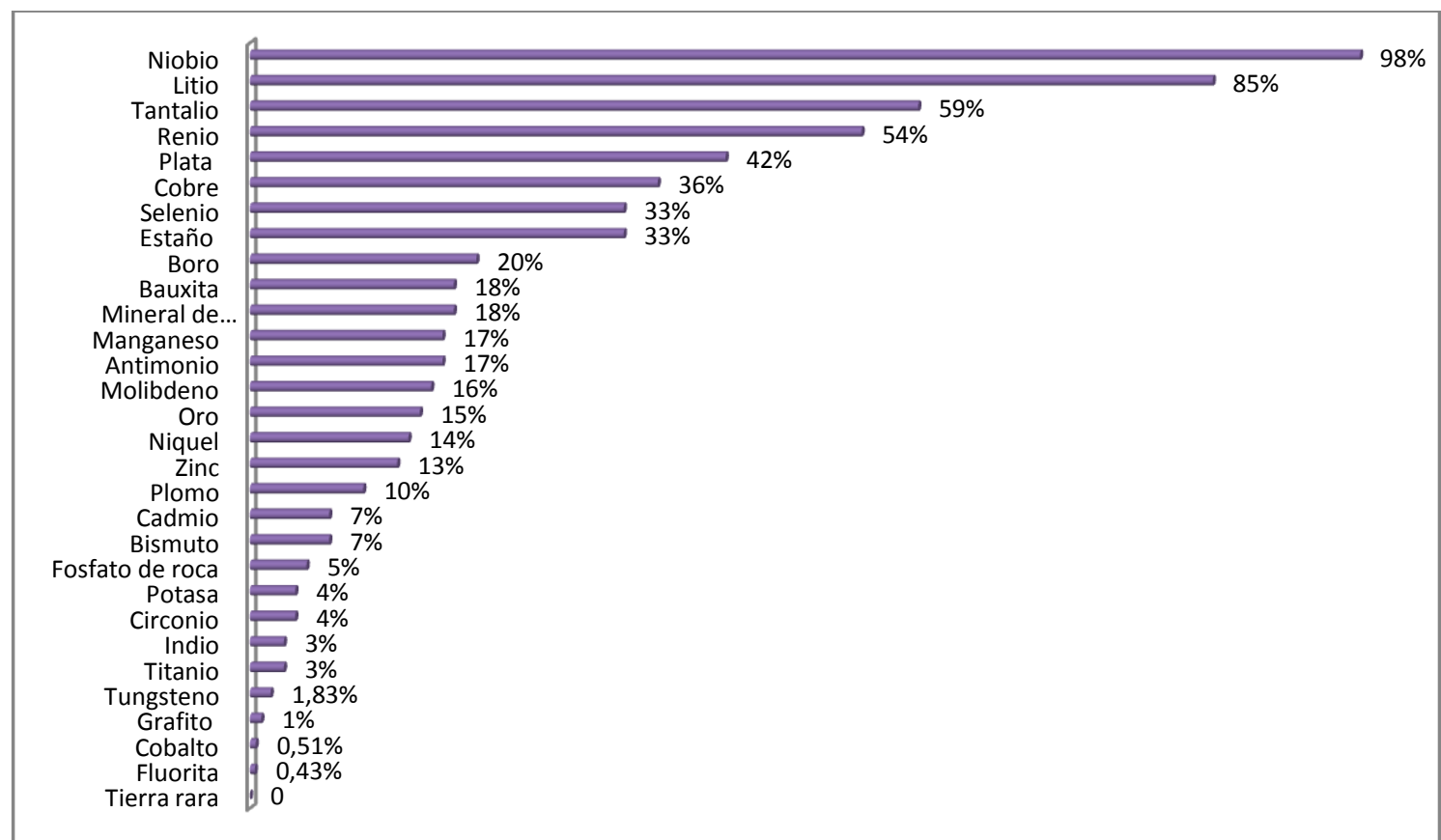

Fuente: OLADE, 2013.

En tercer lugar, en la media que la distribución de minerales en los países de la región esté concentrada en pocos países se favorece las posibilidades de cooperación regional para intervenir en el mercado mundial de la minería en materia de precios.

\section{CUADRO 2: Distribución de los minerales por grupo de países}

\begin{tabular}{|c|c|c|}
\hline Países & Mineral & \% de reservas mundiales \\
\hline Chile, Argentina y Brasil & Litio & $65 \%$ \\
\hline Perú, Chile, Bolivia-México fuera de UNASUR & Plata & $49 \%$ \\
\hline Chile, Perú y México & Cobre & $44 \%$ \\
\hline Perú, Brasil y Bolivia & Estaño & $33 \%$ \\
\hline $\begin{array}{c}\text { Brasil, Guyana, Suriname y Venezuela- Jamaica fuera } \\
\text { de UNASUR }\end{array}$ & Bauxita & $26 \%$ \\
\hline $\begin{array}{l}\text { Brasil, Colombia y Venezuela - Cuba y Rep. } \\
\text { Dominicana fuera de la UNASUR }\end{array}$ & Níquel & $23 \%$ \\
\hline Brasil y Venezuela - México & Hierro & $22 \%$ \\
\hline
\end{tabular}

El grado de concentración de algunos minerales en los países de la región permitiría elaborar pautas de producción que permitan regular el precio en el mercado mundial, especialmente cuando las fluctuaciones de precios oscilen a la baja. Un 


\section{Conjuntura Austral}

ejemplo de ello es la propuesta de crear una "OPEP del Litio" entre Argentina, Bolivia y Chile, sugerida en el 2011 por la Agencia Nacional de Promoción Científica y Tecnológica del Ministerio de Ciencia, Tecnología e Innovación Productiva de Argentina. ${ }^{5}$

Una política de alianzas minerales que entre países sudamericanos puede inclusive complementarse con países por fuera de la región que cuenten con concentración de un mismo mineral. El auge actual de la minería en países africanos presenta una oportunidad para dar mayor sustento a los patrones emergentes de cooperación Sur-Sur, en este caso para aunar criterios de que permitan una intervención en el mercado de minerales para asegurar recursos para impulsar la industrialización y contener la presión a la reprimarización económica.

La UNASUR es una oportunidad para definir estrategias de acción conjunta de intervención en el mercado internacional de minerales. Ello contribuiría a generar condiciones de estabilidad y previsibilidad en los precios, mejorando la capacidad de planificación de políticas de largo alcance en base los ingresos de la minería.

\section{El financiamiento del desarrollo integral}

La administración transparente de la renta minera es condición excluyente para fortalecer las capacidades del estado en el financiamiento de políticas de inclusión social, servicios públicos de calidad e instrumentos que fortalezcan la innovación tecnológica para la industria.

Los recursos naturales son medio para financiar políticas sociales en muchos países del mundo como parte de una concepción integral del desarrollo (Hujo, 2012), aunque también plantean el desafío de la sobrevaluación de sus monedas (enfermedad holandesa). En Sudamérica algunos países han comenzado a experimentar con la creación de fondos soberanos financiados por la renta de los recursos naturales para destinarlos al mejoramiento y expansión de la cobertura servicios públicos. En parte, ello responde a las crecientes demandas ciudadanas por inclusión social y acceso a

${ }^{5}$ http://www.lostiempos.com/diario/actualidad/economia/20110630/argentina-bolivia-y-chile-podriancrear-una-opep-del 131918_267270.html. 


\section{Conjuntura Austral}

servicios públicos - como las protestas por la educación pública en Chile y el transporte en Brasil, etc. La nueva ley minera promulgada en Uruguay incluye un fondo soberano que administrará las ganancias de la minería, además de promover la generación de cadenas de valor y la sustentabilidad ambiental. ${ }^{6}$

La gestión transparente de fondos soberanos, u otros instrumentos de financiación del desarrollo, es fundamental para evitar los conocidos vínculos que se generan con la corrupción, la pobreza e inestabilidad política. Un debate regional sobre buenas prácticas y estándares de transparencia puede tomar como referencia la experiencia del instituto Revenue Watch en la promoción de la gestión transparente de los recursos naturalesen 40 países (que incluyen Bolivia, Brasil, Chile, Colombia, Ecuador y Perú). ${ }^{7}$ Asimismo, la Iniciativa para la Transparencia de las Industrias Extractivas es un antecedente valioso en sus esfuerzos por generar un régimen global de transparencia para industrias extractivas más allá de sus limitaciones actuales (Kantz, 2012).

Además de los distintos arreglos institucionales que promuevan los países, resulta indispensable diseñar sistemas regionales para el financiamiento de políticas de desarrollo. Para ello es central volver impulsar la propuesta de un Banco del Sur, la cual ha perdido gravitación en la agenda regional, mientras que el Banco Nacional de Desarrollo Económico y Social (BNDES) de Brasil se ha convertido en el principal instrumento de financiamiento público en la región (Gomes Saraiva, 2010).

\section{Políticas de inversión minera para el desarrollo integral}

Las perspectivas de que la actividad minera pueda dinamizar otras actividades productivas con el fin de generar valor agregado es de central importancia para lograr los objetivos planteados en la estrategia UNASUR. Para ello, es necesario fortalecer las condiciones de los estados para impulsar encadenamientos productivos dinámicos.

\footnotetext{
${ }^{6}$ http://www.paisminero.co/index.php?option=com_content\&view=article\&id=11946:uruguay-apruebaley-de-megamineria-y-apunta-a-diversificar-su-matriz-productiva\&catid=117:mineriacolombiana\&Itemid $=499$.

${ }^{7}$ http://www.revenuewatch.org/.
} 


\section{Conjuntura Austral}

Las posibilidades de localizar desarrollo tecnológico o capacidades industriales a partir de inversiones mineras de empresas privadas o públicas están ligadas a la capacidad del estado para coordinar actores productivos, empresas y sindicatos, en un marco de políticas de industrialización a largo plazo. Una configuración posible para ello es la generación de aglomeraciones mineras (o clusters) con encadenamientos productivos hacia atrás y adelante. Es decir, con desarrollo de maquinarias, equipamiento e insumos tecnológicos requeridos por la actividad minera, y también procesamiento de minerales y elaboración de semi-manufacturas de origen minero (Buitelaar, 2001). Las políticas de financiamiento de capacidades industriales y tecnológicas son centrales para ello. Además, la integración de cadenas productivas puede, mediante políticas específicas, vincular y contribuir a dinamizar la economía social y solidaria con la integración de cooperativas de producción en la provisión de insumos o servicios (Saguier y Brent 2013).

Estrategias de desarrollo integral a nivel nacional/local pueden también estar coordinadas entre varios países que conforman un mismo clúster sobre la base de un mineral compartido. El "triángulo del litio", comprendido por las zonas de frontera entre Argentina, Bolivia y Chile, es un ejemplo de donde se podría cooperar para un enfoque común en lo relativo a políticas de inversión para la contratación de insumos locales, innovación tecnológica y manufactura con las empresas automotrices y tecnológicas interesadas en el desarrollo de baterías en base al litio. Ejemplos similares de enfoque cooperativos pueden promoverse a partir de otros países que compartan minerales comunes, como los citados anteriormente en el cuadro 2.

La cooperación regional es condición necesaria para impulsar encadenamientos dinámicos en la minería. Sin aunar esfuerzos los estados disponen de menores posibilidades de negociar acuerdos de requisitos de desempeño con las empresas. Inclusive cooperando, los TLCs y los Tratados Bilaterales de Inversión en varios países presentan constreñimientos a los márgenes de negociación. Las empresas utilizan la posibilidad de iniciar demandas en tribunales arbitrales como herramienta de presión. En el mediano plazo pareciera improbable una renegociación de algunas cláusulas o salirse de estos acuerdos. De hecho, la Alianza del Pacífico refuerza el compromiso de 


\section{Conjuntura Austral}

países signatarios de TLCs a profundizar un modelo de inserción internacional basado en el libre mercado. Esto plantea ciertos límites a cuánto pueda la cooperación regional fortalecer el rol del estado en políticas industriales con la minería.

Sin embargo, la evolución política de las agendas y procesos en la UNASUR abren nuevos escenarios que favorecen la construcción capacidades estatales que puedan compensar las restricciones que plantean las diferencias políticas entre países. Ecuador, Venezuela, Bolivia y Ecuador renunciaron al CIADI. Ecuador actualmente promueve la creación de un centro de arbitraje regional de la UNASUR - con instancias de arbitraje, mediación y facilitación. Este proceso tiene implicancias para la minería, en tanto modificaría las condiciones políticas e institucionales que median la relación entre estados y empresas, fortaleciendo así la capacidad de los estados para negociar nuevos acuerdos o renegociar viejos acuerdos de inversión minera con empresas con pautas de desarrollo.

\section{Democratización de las políticas mineras: derecho a consulta y participación social}

La aumento de la actividad minera en la región puso de manifiesto la ausencia de consensos sociales acerca de cuál lugar ocupa la minería, si es que alguno, en las expectativas de desarrollo de los pueblos. La democratización de los procesos de toma de decisión y mecanismos de rendición de cuenta en las políticas de minería para el desarrollo integral es un desafío central dela estrategia UNASUR.

Actualmente en América Latina y el Caribe se registran 196 conflictos en relación a 205 proyectos mineros que afectan 295 comunidades (OCMAL, 2013). Entre los factores recurrentes de tales conflictos esta la ausencia de debate público sobre los cuáles son los supuestos beneficios y costos de la minería, definidos en términos económicos, sociales y ambientales, así de cómo arbitrar equitativamente los derechos y responsabilidades de los principales beneficiarios y damnificados de los mismos. A esto se suma la complicidad de ETNs en casos de violaciones a los derechos humanos de poblaciones que resisten o cuestionan proyectos de industrias extractivas en la región (Saguier 2012a). 


\section{Conjuntura Austral}

En los conflictos sociales sobre la minería se expresan asimismo cuestionamientos a las limitaciones de la concepción imperante del "desarrollo", en tanto crecimiento económico, por considerarla lineal y contraria a las posibilidades de construir una sociedad justa y equilibrada desde una perspectiva social, política, económica y ecológica (De Echave et al., 2009; Escobar, 2010; Svampa y Antonelli, 2009). Conceptos de pueblos indígenas como el buen vivir (Gudynas, 2011; Huanacuni Mamani, 2010) incorporan otras lógicas culturales para entender la relación entre personas y la naturaleza desde miradas socio-ecológicas no reductibles a la racionalidad materialista occidental. Incorporar estas miradas al proceso regional es fundamental para garantizar la construcción democrática del concepto de "desarrollo integral" planteado en la estrategia UNASUR.

El derecho a consulta previa e informada es un instrumento que sirve al reconocimiento de derechos a pueblos indígenas pero también a la democratización de procesos de toma de decisión en lo referido a políticas de recursos naturales. Los países de la UNASUR son firmantes de la Declaración de Naciones Unidas sobre Derechos de los Pueblos Indígenas y algunos de ellos también adhieren y han ratificado el Convenio 169 de la Organización Internacional del Trabajo sobre Pueblos Indígenas y Tribales (Argentina, Estado Plurinacional de Bolivia, Brasil, Chile, Colombia, Ecuador, Paraguay, Perú y República Bolivariana de Venezuela). Perú a partir de 2011 es el primer país en la región en contar con una ley específica de Consulta Previa, Libre e Informada. Bolivia debate actualmente en su parlamento un instrumento normativo similar.

Más allá de los desafíos legislativos, la aplicación del derecho a consulta es fuente de controversia en ambos países. Entre los temas principales se encuentran el cómo deben realizarse las consultaa o si los resultados de las mismas deban ser vinculantes para el estado (KAS, 2012). En Perú, se cuestiona si este derecho se aplicaría a proyectos mineros, mientras que en Bolivia se debate si la ley contemplaría a proyectos de exploración y explotación minera o estaría acotado sólo a la fase de 


\section{Conjuntura Austral}

explotación, entre otras cuestiones. ${ }^{8}$ Colombia, Brasil y Chile, además de Guatemala, México y Panamá, avanzan también procesos legislativos propios.

El tema no obstante excede a los derechos indígenas. Crecientemente comunidades locales demandan su derecho a plebiscitar aquellas decisiones que afectan el hábitat, como es el caso de las actividades mineras y demás industrias extractivas.

La UNASUR es una oportunidad para impulsar, difundir y profundizar la creación de nuevas institucionalidades y prácticas políticas para la consulta previa e informada. Ello es importante en términos de garantizar derechos ciudadanos, pero también como instrumento de política para preservar la paz social y democratizar las políticas mineras. La reciente creación de un Foro de Participación Ciudadana de la UNASUR (VII Cumbre de UNASUR en Surinam, Agosto 2013) abre un espacio consultivo inédito para la participación de organizaciones sociales e indígenas de la región en los procesos de formulación de políticas de integración sudamericana.

\section{Mínimos impactos ambientales negativos}

La incorporación de los más exigentes principios y estándares de protección ambiental, internalización de externalidades negativas ambientales a la actividad minera y de mecanismos de gestión efectivos son tareas indisociables del esfuerzo por generar un marco regional para coordinar políticas de minería para el desarrollo integral.

Como revela la experiencia del Mercosur, los antecedentes de armonización regional de estándares de protección y políticas ambientales no han dejado resultados apremiantes (Hochstetler, 2003). No obstante ello, la politización de la minería, su creciente importancia económica y la oportunidad política que presenta la UNASUR crea condiciones para profundizar un debate sobre principios e instrumentos de regulación ambiental. Ello implica establecer criterios comunes.

En primer lugar, no todos los minerales necesitan ser extraídos. Las propiedades de los minerales para su utilización como insumos industriales son variables. Es factible establecer criterios diferenciadores de minerales en función de su potencial como

${ }^{8}$ http://cedla.org/content/3506. 


\section{Conjuntura Austral}

insumo para industria, y específicamente para los sectores industriales estratégicos que se pretenda promover con políticas de estado. Este criterio restringe el universo de minerales extraíbles, priorizando así los que sean necesarios para la industrialización. El criterio de selección de minerales puede incorporar también otros elementos, como la cantidad de agua y energía requeridas para su extracción y manufactura de modo de priorizar aquellos que redunden en una menor demanda hídrica y energética. El debate sobre principios puede generarse a nivel regional si bien las prioridades y condiciones específicas puedan diferir en cada país.

En segundo lugar, no todos los procesos de exploración y extracción de los minerales son iguales en términos de impacto ambiental. Criterios básicos al respecto deberían incluir la prohibición de uso de sustancias tóxicas utilizadas en actividades mineras (Sacher, 2011), la exigencia de la mejor tecnología disponible, o la adopción de prácticas de gestión de agua adecuadas. Del agua depende la salud humana, el ecosistema y las otras actividades productivas como la agricultura familiar, turismo, etc.

En tercer lugar, no todos los lugares son aptos para la minería. Es necesario definir principios comunes para la zonificación del territorio en función de la vulnerabilidad ecológica. Para ello, es indispensable la adopción de una perspectiva y de las herramientas ambientales estratégicas como el Ordenamiento Ambiental del Territorio. Existen experiencias nacionales en curso que pueden ser compartidas como parte del esfuerzo de cooperación en la UNASUR. Ejemplo de ello es la Ley Nacional de Protección de Glaciares en Argentina del 2010, la primera de su tipo en el mundo. La ley dispone la elaboración de un inventario nacional de glaciares, establece una serie de actividades prohibidas (entre ellas la minería) en el ámbito de afectación de los mismos, como así también la obligación de realizar Evaluaciones de Impactos Ambiental para evitar el daño futuro a glaciares, como así Auditorías Ambientales a los proyectos en ejecución. ${ }^{9}$

\footnotetext{
${ }^{9}$ Uno de los desafíos que debe afrontar esta ley es la resistencia a su aplicación que presentan algunas provincias cordilleranas y la empresa Barrick Gold mediante la judicialización.
} 


\section{Conjuntura Austral}

El debate regional en la UNASUR sobre principios compartidos para la protección del ambiente relacionada la minería es de relevancia estratégica para los países. Por un lado porque este debate permitiría adecuar legislaciones y políticas existentes o nuevas en base a principios de protección más exigente, cuando este fuera el caso. Por otro lado, porque los estados podrían así generar un parámetro básico común que contribuya a evitar la competencia regulatoria entre países en materia de regulación ambiental.

La regionalización de experiencias y estándares de gestión de los procesos de evaluación de impacto ambiental es central. Para ello se pueden conformar redes de equipos especializados integrados por administradores públicos en ámbitos de gestión ambiental, institutos de investigación científica, universidades y organizaciones de la sociedad civil. Este tipo de comunidades epistémicas, o redes de política pública, debería contar con un financiamiento estable y con funciones coordinadas por un grupo de trabajo integrado por las distintas comisiones de la UNASUR abocadas a políticas relacionadas a recursos naturales: Consejo de Infraestructura y Planeamiento (COSIPLAN), Consejo Energético, Consejo de Ciencia, Tecnología e Innovación y el propuesto Instituto Geológico de la UNASUR.

\section{Conclusión}

El proceso UNASUR puede potencialmente contribuir a catalizar un debate político regional para la definición de principios comunes para una estrategia sobre recursos naturales para el desarrollo integral. Ello depende de cuales sean los temas de agenda que orienten este debate y esfuerzos de cooperación regional. Las áreas de política sugeridas plantean importantes desafíos para los gobiernos. Ninguna de ellas puede prescindir de las otras, si lo que está en cuestión es vincular una estrategia regional de políticas de minería para la construcción de agendas e instrumentos de desarrollo integral.

No obstante ello, la complejidad de temas y divergencia de intereses $\mathrm{y}$ percepciones entre países hace que las posibilidades de alcanzar una estrategia general a nivel UNASUR sobre todos los puntos sugeridos parezcan altamente improbables. 


\section{Conjuntura Austral}

Frente a ello, el artículo propone modestamente estimular el debate, entendiendo que se trata de un proceso político en construcción, abierto, dinámico y de largo plazo.

Se derivan algunas conclusiones preliminares. En primer lugar, la competencia regulatoria (en materia fiscal, ambiental, social) puede detenerse con mayores probabilidades con políticas cooperativas en algunos metales como el litio, la plata y el cobre en los que hay pocos países involucrados. En la medida que la distribución de minerales sea más dispersa geográficamente, se dificultaría la acción colectiva por la mayor cantidad de actores involucrados. Además de contener la competencia regulatoria, la cooperación en base a minerales compartidos plantea posibilidades de formular políticas activas y concertadas en materia regulatoria, de precios (especialmente cuando empresas públicas tienen participación en la producción) y políticas de inversión que dinamicen el desarrollo industrial. Ello supone nuevos compromisos en acuerdos entre estados y empresas.

En segundo lugar, los países con empresas mineras públicas cuentan con mayores elementos para la negociación con empresas privadas. Asimismo, la creciente presencia de empresas públicas en la región compensa a países que cuenten con menores posibilidades tecnológicas propias, aunque también genera asimetrías internas entre los países. El carácter público, o mayoritariamente público, de las empresas no es garantía de que éstas tengan proyectos de inversión diferentes a las privadas. La cooperación entre empresas mineras públicas en el marco de estrategias de desarrollo es condición necesaria para realizar los objetivos planteados en la UNASUR.

En tercer lugar, las crecientes demandas ciudadanas por participación y derecho a consulta previa e informada en las políticas de minería (y demás recursos naturales) genera condiciones para elaborar nuevos marcos institucionales y políticas para democratizar los procesos de toma de decisión e implementar políticas de minería. El Foro de Participación Ciudadana en la UNASUR brinda la oportunidad de que gobiernos incorporen en el proceso regional demandas y aportes de organizaciones sociales que ya articulan regionalmente demandas ciudadanas en coaliciones transnacionales, y por lo tanto consensos compartidos entre diversos sectores sociales vinculados a la minería. La regionalización de nuevos mecanismos nacionales y 


\section{Conjuntura Austral}

regionales de participación social constituye una dimensión estratégica de la política de fortalecimiento de la UNASUR.

Las posibilidades de aumentar la autonomía política de los países sudamericanos en la construcción de sus modelos de desarrollo, y en la definición de los términos de inserción en la globalización, requieren más que nunca de la coordinación como bloque regional en materia de recursos naturales. El contenido y alcance de una política regional de minerales estarán sin duda supeditados a las posibilidades políticas concretas de cada país, además de las orientaciones ideológicas y posicionamientos internacionales de los mismos. Sin embargo, la incorporación de la agenda de recursos naturales y desarrollo integral al ámbito de la UNASUR plantea por primera vez la oportunidad política para un debate amplio y regional.

Mientras antes podamos avanzar sobre nuevos consensos en materia de recursos naturales, mejor estaremos preparados para avanzar en la profundización de nuevas bases de soberanía. Esto demandará reinventar instrumentos y prácticas de intervención política para la generación de nuevos acuerdos y mecanismos regulación para las empresas y para la representación de intereses sociales. De lo contrario, la estrategia UNASUR sobre recursos naturales perderá relevancia y gravitación política. Peor aún, representará una forma de cooperación regional para viabilizar coordinadamente el saqueo de los minerales. 


\section{Conjuntura Austral}

\section{REFERÊNCIAS}

BEBBINGTON, Anthony (ed.). Social Conflict, Economic Development and Extractive Industry in Latin America. Abington/New York: Routledge, 2012.

BUITELAAR, Rudolf M. (ed.). Aglomeraciones Mineras y Desarrollo Local en América Latina. Bogotá: CEPAL/Alfaomega Grupo Editor, 2001.

CAOI. El derecho a la consulta y al consentimiento previo, libre e informado. Avances, limitaciones $\mathbf{y}$ retos. Informe Regional, Coordinadora Andina de Organizaciones Indígenas, 2012 [http://es.scribd.com/doc/130202231/Libro-Consultaen-PDF-Copy-pdf].

CEPAL. Recursos Naturales en UNASUR: Situación y tendencias para una agenda de desarrollo regional. LV/L. 3627, Mayo.Chile: Naciones Unidas, 2013, pp. 1-110 [http://www.eclac.org/publicaciones/xml/3/49893/RecursosNaturalesUNASUR.pdf].

CUTLER, Clair. Private international regimes and interfirm cooperation.HALL, Rodney and BIERSTEKER, Thomas (ed.). The Emergence of Private Authority in Global Governance.Cambridge: Cambridge UniversityPress, 2003.

DE ECHAVE, José C.; Raphael HOETMER, and Mario Palacios PANÉZ (ed.).Minería y Territorio en el Perú. Lima: Programa de Democracia y Transformación Global, CONACAMI, CooperAcción, UNMSM, 2009.

ESCOBAR, Arturo. Latin America at the crossroads.Cultural Studies, vol. 24, no. 1, 2010, pp. 1-65.

FUCHS, Doris A. The Role of Business in Global Governance. Schirm, S.A. (ed.). New Rules for Global Markets Public and Private Governance in the World Economy. London: Palgrave Macmillan, 2004, pp. 133-154.

GOMES SARAIVA, Myriam. Brazilian foreign policy towards South America during the Lula Administration: caught between South America and Mercosur. Revista Brasileira de Politica Internacional, vol. 53 (special edition), 2010. pp. 151-168.

GUDYNAS, Eduardo. Estado compensador y nuevos extractivismos. Las ambivalencias del progresismo sudamericano. Nueva Sociedad, vol. 237, 2012. Pp. 128-146.

Buen vivir: Germinando alternativas al desarrollo. América Latina en Movimiento - ALAI, vol. 462,2011. Pp. 1-20. 


\section{Conjuntura Austral}

HEIDRICH, Pablo. Tax Regimes on Mining in Latin America. Policy Brief, North South Institute, Ottawa, 2013.

HALL, Rodney and BIERSTEKER, Thomas. The Emergence of Private Authority in Global Governance. Cambridge: Cambridge University Press, 2003.

HOCHSTETLER, Katherine.Fading Green? Environmental Politics in the Mercosur Free Trade Agreement. Latin American Politics and Society, vol. 45, no. 4, 2003. Pp. $1-32$.

HOGENBOOM, Barbara. Depolitiziced and Repoliticized Minerals in Latin America. Journal of Developing Societies, vol. 28, 2012. Pp. 133-158.

HUJO, Katja (ed.). Mineral Rents and the Financing of Social Policy: Opportunities and Challenges. Basingstoke: UNRISID/PalgraveMacmillan, 2012.

HUANACUNI MAMANI. Buen Vivir/Vivir Bien: Filosofía, políticas, estrategias y experiencias regionales andinas. Lima: CoordinadoraAndina de OrganizacionesIndígenas - CAOI, 2010.

KECK, Margaret and SIKKINK, Katherine.Activists Beyond Borders: Advocacy Networks in International Politics. Ithaca, NY: Cornell University Press, 1998.

MINING PRESS, No. 53, Año 9, 2013.

KANZ, Carola. The Extractive Industries Transparency Initiative (EITI).REED,D., UTTING,P. and MUKHERJEE REED, A. (ed.): Business, Non-State Regulation and Development: Whose Standards? Whose Development? Routledge: London, 2012. Pp. 225-238.

KAS. El derecho a consulta previa de los pueblos indígenas de América latina. Fundación Konrad Adenauer, La Paz, 2012 [http://www.kas.de/wf/doc/kas 335921522-4-30.pdf?130221162840].

KEOHANE, Robert O. and Nye, Joseph S. Governance in a globalizing world. KEOHANE, R.O. (ed.): Power and governance in a partially globalized world. Suffolk: Routledge, 2002.

OCMAL. Base de datos conflictos mineros. Observatorio de Conflictos Mineros de América Latina [http://www.conflictosmineros.net/].

OLADE Potencial de Recursos Energéticos y Minerales en América del Sur Coincidencias Jurídicas hacia una Estrategia Regional. Organización Latinoamericana de Energía. Quito, 2013. Pp. 1-140. 


\section{Conjuntura Austral}

RIGGIROZZI, Piaand TUSSIE, Diana (ed.). The Rise of Post-Hegemonic Regionalism: The Case of Latin America. Dordrecht/Heidelberg/London/New York: Springer, 2012.

RODRÍGUEZ-GARAVITO, César, Patrick BARRETT and Daniel CHÁVEZ.Utopia reborn? Introduction to the study of the new Latin American left. BARRETT. P., CHÁVEZ, D.and RODRÍGUEZ-GARAVITO, C., (ed.): The new Latin American left: Utopia reborn? London: Pluto Press, 2008.

SACHER, William. La Cara Tóxica del Oro - Una introducción al uso del cianuro en la explotación del oro. Santiago: Editorial Quimantu, 2011 [http://es.scribd.com/doc/54235978/Cianuro-cara-Toxica-Del-Oro].

SAGUIER, Marcelo. Peoples' Tribunals in Latin America.REED, D., UTTING, P. and MUKHERJEE REED, A. (ed.): Business, Non-State Regulation and Development: Whose Standards? Whose Development? Routledge: London, 2012a. Pp. 225-238.

Socio-environmental regionalism in South America: tensions in the new development models. RIGGIROZZI, P.and TUSSIE, D. (ed.): The Rise of PostHegemonic Regionalism: The Case of Latin America. Dordrecht/Heidelberg/London/New York: Springer, 2012b. pp. 125-145.

SAGUIER, Marcelo y BRENT, Zoe. Regional Policy Frameworks for Social and Solidarity Economy in South America. UNRISD Conference: Potential and Limits of Social and Solidarity Economy, United Nations Institute for Social Development, Geneva, 6-8 May, 2013.

SASSEN, Saskia. Territory, Authority, Rights: From Medieval to Global Assemblages. Princeton: Princeton UniversityPress, 2006.

SVAMPA, Maristella y ANTONELLI Mirta A. (ed.). Minería Transnacional, Narrativas del Desarrollo y Resistencias Sociales. Buenos Aires: Biblos, 2009.

TARROW, Sydney. The New Transnational Activism. Cambridge: Cambridge University Press, 2005.

UTTING, Peter. Social and Environmental Liabilities of Transnational Corporations New Directions, Opportunities, and Constraints.UTTING, Peter and CLAPP, Utting, P. and Clapp, J. (ed.): Corporate Accountability and Sustainable Development. New Delhi: OUP, 2008. 


\title{
Conjuntura Austral
}

\section{RESUMEN}

El artículo analiza las condiciones en las que la UNASUR podría facilitar una estrategia regional de aprovechamiento de la minería para el desarrollo integral. Ello depende de que logre fortalecer las capacidades delos estados de intervenir en el mercado de minerales, regular la minería y democratizar las políticas mineras.

\section{PALABRAS CLAVE}

UNASUR; minería; desarrollo.

\section{RESUMO}

O artigo analisa as condiçõesem que a UNASUL poderia facilitar umaestratégia regional para o uso da mineração para o desenvolvimento. Depende de quemconseguereforçar as capacidades dos países para intervir no mercado de minerais, regular a mineração e democratizar as políticas de mineração.

\section{PALAVRAS-CHAVE}

UNASUL, mineração, desenvolvimento.

\begin{abstract}
The article analyzes the conditions under which the UNASUR could facilitate a regional strategy for the use of mining for development. Necessary conditions for this are the strengthening of states' capacities of states to intervene in the minerals, to regulate its activity and democratize mining policies.
\end{abstract}

\section{KEYWORDS}

UNASUR, mining, development. 